\title{
Minimal Hepatic Encephalopathy and Biejia-Ruangan Are Associated with First Hospital Readmission in Nonalcoholic Cirrhosis Patients
}

\author{
Ting-Ting Jiang, Xiao-Li Liu, Yu-Yong Jiang $\mathbb{D}^{\mathbb{D}}$, Xian-Bo Wang $\mathbb{D}$, and Zhi-Yun Yang \\ Center of Integrative Medicine, Beijing Ditan Hospital, Capital Medical University, Beijing 100015, China \\ Correspondence should be addressed to Yu-Yong Jiang; jyuy11@126.com, Xian-Bo Wang; wangxianbo638@163.com, and \\ Zhi-Yun Yang; yangzhiyun2016@163.com
}

Received 27 December 2020; Revised 6 April 2021; Accepted 19 April 2021; Published 8 May 2021

Academic Editor: Guang Chen

Copyright ( 2021 Ting-Ting Jiang et al. This is an open access article distributed under the Creative Commons Attribution License, which permits unrestricted use, distribution, and reproduction in any medium, provided the original work is properly cited.

\begin{abstract}
Introductionand Aim. Patients with cirrhosis are often hospitalized repeatedly for a variety of complications. This retrospective study aimed to assess the effects of minimal hepatic encephalopathy (MHE) and Biejia-Ruangan (BR) on first hospital readmission in nonalcoholic cirrhosis patients without previous overt hepatic encephalopathy (OHE) or hepatocellular carcinoma (HCC). Materials and Methods. A total of 176 hospitalized patients with nonalcoholic cirrhosis were included in this retrospective study. Patients who were first admitted to Beijing Ditan Hospital of Capital Medical University from January 2017 to September 2019 were enrolled. The primary endpoint was their first liver-related hospital readmission. The risk factors for readmission were analyzed by Cox proportional hazard regression analysis. Results. A total of 176 nonalcoholic cirrhosis patients without previous OHE or HCC were included; 57 patients (32.4\%) were diagnosed with MHE, and 63 patients (35.8\%) were administered BR (2 g, three times a day). Multivariate analysis revealed that nonalcoholic cirrhosis patients with MHE (HR, 5.805; 95\% CI, 3.007-11.206; $x, P<0.001)$ and a higher Model for End-Stage Liver Disease (MELD) score (HR, 1.145; 95\% CI, 1.068-1.227; $P<0.001)$ had an increased risk of first hospital readmission, and patients treated with BR (HR, 0.318; 95\% CI, 0.151-0.670; $P=0.003$ ) had a decreased risk of first hospital readmission. Conclusion. MHE increased the risk of hospital readmission in nonalcoholic cirrhosis patients without previous OHE or HCC, and this risk was decreased by BR administration.
\end{abstract}

\section{Introduction}

Patients with cirrhosis are often readmitted to the hospital because of various complications. One of the most serious complications is hepatic encephalopathy (HE). Minimal hepatic encephalopathy (MHE), which is the mildest form on the hepatic encephalopathy spectrum, is usually not easily detected. There are no typical neurological and psychiatric manifestations in MHE patients, and MHE can only be detected through neuropsychological or neurophysiological tests [1]. Patients with MHE appear normal, while there are hidden dangers associated with driving and falling that affect their quality of life (QOL) [2-5]. Worldwide, the prevalence of MHE ranges from $30 \%$ to $84 \%$ in patients with cirrhosis [6-8]. Neurophysiological tests take a substantial amount of time, and clinicians are often too busy to screen patients with cirrhosis $[9,10]$. In recent years, some studies have shown that the clinical prognosis of MHE patients is worse than that of cirrhosis patients with normal neuropsychological test results, and the mortality of MHE patients is significantly elevated due to hepatocellular carcinoma (HCC) and liverrelated complications [11-13]. During clinical follow-up, a large proportion of MHE patients develop overt hepatic encephalopathy (OHE) and other complications. Once severe hepatic encephalopathy occurs, the one-year survival rate is less than $50 \%$, and the three-year survival rate is less than $25 \%$ [14].

Some studies have revealed that smoking, the Child-Pugh score (CTP), the Model for End-Stage Liver Disease (MELD) score, the serum albumin (ALB) level, and the ammonia level are associated with the presence of MHE $[11,15,16]$. Other studies reported that age, gender, albumin 
level, HCC stage, MHE, CTP, and MELD scores were related to mortality $[17,18]$. However, those studies always involved patients with alcoholic cirrhosis, previous OHE, and/or HCC, which may have affected the clinical outcomes [19-21]. Therefore, we rigorously excluded patients with alcoholic cirrhosis, previous OHE, or HCC in our study.

Traditional Chinese medicine (TCM), as an ancient medical system, has been applied worldwide [22]. Clinical trials have demonstrated that BR can block the development of hepatic fibrosis and reverse early cirrhosis [23, 24]. According to TCM theory, the pathogenesis of cirrhosis is weakened qi and blood, accompanied by blood stasis [25]. Biejia-Ruangan (BR), which is composed of at least 32 antifibrotic compounds, was formulated to promote qi and blood and invigorate the blood circulation to remove blood stasis [26]. The China Food and Drug Administration (CFDA) approved BR (Inner Mongolia Furui Medical Science Co Ltd, Wulanchabu, China, license number Z1999101) as the first TCM antifibrotic regimen to treat cirrhosis caused by chronic hepatitis B (CHB).

This study was carried out to determine the risk factors for the diagnosis and prognosis of MHE in nonalcoholic cirrhosis patients without previous $\mathrm{OHE}$ or HCC and to evaluate the effect of $\mathrm{BR}$ on the prognosis of these cirrhosis patients.

\section{Materials and Methods}

2.1. Study Design and Patients. In this retrospective study, we enrolled a total of 237 nonalcoholic cirrhosis patients who had been first admitted to our hospital between January 2017 and September 2019. Of these patients, 61 were excluded due to the following reasons: HCC $(N=16)$, OHE or a history of OHE $(N=3)$, drug use $(N=14)$, fever $(N=3)$, alcohol abuse $(N=20)$, vision problems, or refusal to undergo the test $(N=5)$. A total of 176 patients met the eligibility criteria. All patients meeting the criteria were included.

The inclusion criteria were as follows: hospitalized nonalcoholic cirrhosis patients between 20 and 75 years of age who underwent testing for MHE according to the psychometric hepatic encephalopathy score (PHES). The exclusion criteria were as follows: (1) the presence of OHE or a history of OHE; (2) a history of alcohol abuse; (3) the presence of HCC; (4) infection or spontaneous bacterial peritonitis (SBP) in the past 4 weeks; (5) gastrointestinal bleeding in the past 4 weeks; (6) the presence of neurological diseases; (7) the presence of psychiatric disorders or severe comorbidities; (8) use of drugs when the tests were performed, including benzodiazepines, antiepileptic or psychotropic substances, probiotics, rifaximin, and ammonia-lowering drugs (L-ornithine-L-aspartate, lactulose, and lactitol); (9) inability to undergo testing due to a lack of education or vision problems such as glaucoma, cataract, or other reasons; (10) a history of shunt surgery or the insertion of a transjugular intrahepatic portosystemic shunt; (11) use of TCM treatments other than BR; and (12) incomplete clinical data.

BR consists of more than 32 antifibrotic compounds [24]. The major ingredients of BR are shown in Table 1.

The definition of BR therapy (BR users) was as follows: medical records of BR use (a dosage of $2 \mathrm{~g}$ of $\mathrm{BR}$ three times a
TABLE 1: The major ingredients of Biejia-Ruangan (BR).

\begin{tabular}{lc}
\hline Chinese phonetic alphabet name & English name \\
\hline Bejia & Turtle shell \\
Chishao & Peony root \\
Ezhu & Zedoray rhizome \\
Danggui & Angelica sinensis \\
Dangshen & Campanumaea pilosula \\
Huangqi & Astragalus \\
Sanqi & Pseudo-ginseng \\
Dongchongxiacao & Plant worms \\
Ziheche & Dried human placenta \\
Banlangen & Baphicacanthus root \\
Lianqiao & Farsythio \\
\hline
\end{tabular}

day for at least seven days during hospitalization and at least a total of six months of BR administration after discharge from the hospital).

The definition of BR-6 therapy was as follows: a duration of $\mathrm{BR}$ therapy of six months

The definition of BR-12 therapy was as follows: a duration of $\mathrm{BR}$ therapy of twelve months

2.2. Diagnosis of Minimal Hepatic Encephalopathy. All enrolled patients underwent PHES assessment, which consists of five subtests, namely, number connection tests (NCTs) A and $\mathrm{B}$, a digit symbol test (DST), a line tracing test (LTT), and a serial dotting test (SDT). These tests were administered by well-trained specialists. Patients with liver cirrhosis were diagnosed with MHE if the results of more than two subtests were abnormal $[27,28]$.

2.3. Outcomes. The data were tracked via our electronic medical system with confirmation via telephone calls made by the researcher. The time from the initial inpatient screening to the first liver-related rehospitalization was recorded in months. Hospitalizations due to periodic evaluation were not included. Liver-related rehospitalizations were defined as those related to cirrhosis complications (hepatorenal syndrome, variceal bleeding, ascites and related spontaneous bacterial peritonitis, encephalopathy, and jaundice) and HCC. The primary endpoint was the first liver-related rehospitalization.

2.4. Clinical Data Collection. Cirrhosis was defined by ultrasound, CT scan, MRI, endoscopic analysis, biochemical parameters, and liver biopsy, if available. Liver dysfunction was evaluated by the MELD scores and the CTP. Blood samples were obtained from patients within 7 days of the MHE test. Demographic and laboratory parameters were collected for all patients from the electronic medical system, including gender, age, white blood cell (WBC) count, hemoglobin (HGB) level, thrombocyte (PLT) count, alanine transaminase (ALT) level, aspartate aminotransferase (AST) level, total bilirubin (TBIL) level, serum albumin (ALB) level, creatinine $(\mathrm{Cr})$ level, international normalized ratio (INR), potassium (K) level, and sodium (Na) level. The 
etiology of cirrhosis was categorized into hepatitis B infection, hepatitis $\mathrm{C}$ infection, autoimmune-associated cirrhosis, and other cryptogenic cirrhosis. Compensated cirrhosis was defined as cirrhosis without complications. Decompensated cirrhosis was defined as cirrhosis with complications such as hepatorenal syndrome, variceal bleeding, ascites and related spontaneous bacterial peritonitis, encephalopathy, and jaundice.

All cirrhotic patients were treated according to the European Association for the Study of the Liver (EASL) clinical practice guidelines [29-32]. All procedures performed in studies involving human participants were in accordance with the ethical standards of the institutional and/or national research committee and with the 1975 Helsinki declaration and its later amendments or comparable ethical standards.

2.5. Statistical Analysis. Statistical analysis was performed using IBM SPSS version 23 (IBM Corp, NY) and Stata (Stata Corp LLC). Normally distributed continuous data were expressed as the mean \pm standard deviation (SD) and were analyzed by Student's $t$-tests. Nonnormally distributed continuous data were presented as the median and interquartile range (IQR) and were analyzed using the Mann-Whitney U test. Categorical data were expressed as frequencies and were analyzed using the chi-square test and Fisher's exact test. The risk factors for MHE were analyzed by logistic regression analyses. The risk factors of first hospital readmission were analyzed by multivariate Cox regression analyses. Rehospitalization risk factors were analyzed by the Kaplan-Meier method and the log-rank test. Cut-offs for continuous variables were based on the Youden index. Two-sided $P$ values $<0.05$ were considered statistically significant.

\section{Results}

3.1. Baseline Characteristics. In total, there were 176 consecutive patients, including 103 men and 73 women (age range, $24-73$ years; $51.1 \pm 10.7)$. The etiology of liver cirrhosis included hepatitis B virus infection $(N=136)$, hepatitis C virus infection $(N=14)$, autoimmune hepatitis $(n=17)$, and cryptogenic cirrhosis $(N=9)$. The numbers of cirrhotic patients in Child-Pugh classes A, B, and C were 126 (71.6\%), 42 (23.9\%), and 8 (4.5\%), respectively. No significant difference was found between the BR group and no-BR group $(P>0.05)$ (Table 2).

3.2. Factors Associated with Minimal Hepatic Encephalopathy among the 176 Nonalcoholic Cirrhosis Patients. Among the 176 eligible patients, 57 (32.4\%) were diagnosed with MHE. The prevalence rates of MHE in patients in Child-Pugh classes A, B, and C were 33 of 126 (26.2\%), 18 of 42 (42.9\%), and 6 of 8 (75\%), respectively. In the univariate analysis, MHE was closely associated with serum albumin level, serum $\mathrm{Na}$ level, serum $\mathrm{K}$ level, and $\mathrm{BR}$ use. A logistic regression analysis was performed using age, ALT, AST, INR, MELD, serum Cr, ALB level, TBIL, serum Na level, serum K level, WBC count, and BR as variables. Only the ALB level was significantly associated with the presence of MHE (HR, 0.92; 95\% CI, 0.852-0.993; $P=0.032$ ) (Table 3). A decreased ALB level was related to a greater risk of MHE in nonalcoholic cirrhosis patients.

\subsection{Factors Associated with First Hospital Readmission among} the 176 Nonalcoholic Cirrhosis Patients. Patients were followed for a median of 17 months (IQR, 5.3, 26.8). During the study period, 45 (25.6\%) patients were first readmitted. In total, four patients died during their first readmission; two patients died of jaundice, one patient died of hepatorenal syndrome, and another patient died of ascites and related spontaneous peritonitis. The first hospital readmission events included 5 cases of encephalopathy, 5 cases of HCC, 17 cases of ascites and related spontaneous peritonitis, 14 cases of gastrointestinal bleeding, 3 cases of jaundice, and 1 case of hepatorenal syndrome.

The prognostic factors in nonalcoholic cirrhotic patients are summarized in Table 4. Univariate Cox regression analysis revealed that MHE, BR use, the MELD score, serum Na level, ALB level, and TBIL level were risk factors for first hospital readmission. The multivariate analysis identified nonalcoholic cirrhotic patients with MHE (HR, 5.805; 95\% CI, 3.007-11.206; $P<0.001)$ and a higher MELD score (HR, 1.145; 95\% CI, $1.068-1.227 ; P<0.001$ ) as associated with a poor prognosis and patients treated with $\mathrm{BR}$ (HR, 0.318; 95\% CI, 0.151-0.670; $P=0.003$ ) as having a favorable prognosis (Table 4). The cut-off value (specificity + sensitivity-1) for the MELD score was 15 . There was marked difference in first hospital readmission risk between the MELD $\geq 15$ group and the MELD $<15$ group $(P<0.001)$ (Figure 1).

3.4. MHE and First Hospital Readmission in the 176 Nonalcoholic Cirrhosis Patients. The first hospital readmission events in the MHE group included 4 cases of encephalopathy, 2 cases of HCC, 14 cases of ascites and related spontaneous peritonitis, 8 cases of gastrointestinal bleeding, 3 cases of jaundice, and 1 case of hepatorenal syndrome. In the no-MHE group, there were 3 cases of HCC, 3 cases of ascites and related spontaneous peritonitis, 6 cases of gastrointestinal bleeding, and 1 case of encephalopathy. When all first hospital readmission events during the follow-up were considered, there were significantly more events in the MHE group, with 32 events $(0.56$ events per patient) vs. 13 events in the no-MHE group (0.11 events per patient $)(P<0.001)$. The cumulative incidence of first hospital readmission was significantly higher in the MHE group than in the no-MHE group $(P<0.001)$ (Figure 2).

3.5. BR and First Hospital Readmission in the 176 Nonalcoholic Cirrhosis Patients. The first hospital readmission events in the BR group included 1 case of HCC, 3 cases of ascites and related spontaneous peritonitis, 3 cases of gastrointestinal bleeding, and 2 cases of encephalopathy. The first hospital 
TABLE 2: Clinical characteristics of patients with and without Biejia-Ruangan (BR) treatment in nonalcoholic cirrhosis.

\begin{tabular}{|c|c|c|c|}
\hline & \multicolumn{3}{|c|}{ All patients $(N=176)$} \\
\hline & $\mathrm{BR}(N=63)$ & No-BR $(N=113)$ & $P$ value \\
\hline Age, mean \pm SD (range) (yr) & $50.8 \pm 11.1$ & $52.3 \pm 10.3$ & 0.745 \\
\hline Gender $(\mathrm{m} / \mathrm{f})$ & $37 / 26$ & $66 / 47$ & 0.967 \\
\hline Etiology of cirrhosis & & & 0.794 \\
\hline Hepatitis B & 49 & 87 & \\
\hline Hepatitis C & 6 & 8 & \\
\hline Autoimmune hepatitis & 6 & 11 & \\
\hline Unknown & 2 & 7 & \\
\hline Serum albumin $(\mathrm{g} / \mathrm{L})$ & $35.5 \pm 5.1$ & $35.8 \pm 5.8$ & 0.090 \\
\hline Decompensated cirrhosis & $45 / 18$ & $87 / 26$ & 0.414 \\
\hline $\mathrm{K}+(\mathrm{mmol} / \mathrm{L})$ & $3.82 \pm 0.39$ & $3.95 \pm 0.37$ & 0.133 \\
\hline Child-Pugh class & & & 0.967 \\
\hline A & 45 & 81 & \\
\hline B & 15 & 27 & \\
\hline $\mathrm{C}$ & 3 & 5 & \\
\hline $\operatorname{ALT}(\mathrm{U} / \mathrm{L})$ & $55.5(29.2,21.9)$ & $42.5(23.5,17.2)$ & 0.069 \\
\hline $\operatorname{AST}(\mathrm{U} / \mathrm{L})$ & $54.5(36.9,39.9)$ & $58.4(28.8,18.9)$ & 0.084 \\
\hline Total bilirubin (mg/dL) & $32.4(21.5,21.6)$ & $33.8(19.5,14.3)$ & 0.408 \\
\hline $\mathrm{Na}+(\mathrm{mmol} / \mathrm{L})$ & $140.7 \pm 2.9$ & $140.0 \pm 3.6$ & 0.716 \\
\hline INR & $1.26(1.23,0.25)$ & $1.30(1.21,0.33)$ & 0.982 \\
\hline Creatinine $(\mu \mathrm{mol} / \mathrm{L})$ & $64.7(57.2,20.5)$ & $64.9(63.6,17.2)$ & 0.130 \\
\hline Hemoglobin $(g / L)$ & $121.9 \pm 24.9$ & $113.1 \pm 23.2$ & 0.396 \\
\hline Platelet $\left(10^{9} / \mathrm{L}\right)$ & $92.8(73.3,76)$ & $85.6(60.0,63.0)$ & 0.526 \\
\hline White blood cells $\left(10^{9} / \mathrm{L}\right)$ & $3.53(3.15,2.55)$ & $3.53(3.28,1.84)$ & 0.704 \\
\hline MELD & $10.9(10.0,5.0)$ & $10.6(9,4)$ & 0.389 \\
\hline
\end{tabular}

Abbreviations: K+, potassium; $\mathrm{Na}+$, sodium; ALT, alanine aminotransferase; AST, aspartate aminotransferase; INR, international normalized ratio; MELD, model for end-stage liver disease; BR, Biejia-Ruangan.

TABLE 3: Univariate and multivariate regression analysis for predictors of minimal hepatic encephalopathy (MHE) in nonalcoholic cirrhosis patients $(N=176)$.

\begin{tabular}{|c|c|c|c|c|c|c|c|c|}
\hline \multirow[t]{2}{*}{ Variable } & \multirow[t]{2}{*}{ OR } & \multicolumn{2}{|c|}{$\begin{array}{l}\text { 95\% variable OR CI } \\
\text { for EXP (B) }\end{array}$} & \multirow[t]{2}{*}{$P$ value } & \multirow[t]{2}{*}{ OR } & \multicolumn{2}{|c|}{$\begin{array}{l}95 \% \text { variable OR CI } \\
\text { for EXP (B) }\end{array}$} & \multirow[t]{2}{*}{$P$ value } \\
\hline & & Lower & Upper & & & Lower & Upper & \\
\hline Age (year) & 1.109 & 0.989 & 1.050 & 0.214 & 1.031 & 0.995 & 1.069 & 0.095 \\
\hline $\operatorname{ALT}(\mathrm{U} / \mathrm{L})$ & 1 & 0.997 & 1.003 & 0.941 & 0.987 & 0.965 & 1.010 & 0.260 \\
\hline AST & 1.001 & 0.998 & 1.003 & 0.475 & 1.010 & 0.994 & 1.027 & 0.205 \\
\hline $\mathrm{Na}+(\mathrm{mmol} / \mathrm{L})$ & 0.893 & 0.802 & 0.994 & 0.039 & 0.924 & 0.815 & 1.047 & 0.215 \\
\hline $\mathrm{K}+(\mathrm{mmol} / \mathrm{L})$ & 2.744 & 1.121 & 6.716 & 0.027 & 2.496 & 0.923 & 6.749 & 0.072 \\
\hline Serum albumin $(\mathrm{g} / \mathrm{L})$ & 0.925 & 0.872 & 0.982 & 0.010 & 0.920 & 0.852 & 0.993 & 0.032 \\
\hline Total bilirubin $(\mu \mathrm{mol} / \mathrm{L})$ & 1.006 & 0.998 & 1.015 & 0.130 & 1.004 & 0.990 & 1.018 & 0.592 \\
\hline INR & 0.511 & 0.139 & 1.874 & 0.311 & 0.580 & 0.141 & 2.383 & 0.450 \\
\hline Creatinine $(\mu \mathrm{mol} / \mathrm{L})$ & 1.002 & 0.989 & 1.015 & 0.783 & 1.002 & 0.986 & 1.019 & 0.801 \\
\hline White blood cells $\left(10^{9} / \mathrm{L}\right)$ & 0.943 & 0.774 & 1.15 & 0.563 & 0.926 & 0.735 & 1.166 & 0.514 \\
\hline $\mathrm{BR}$ & 0.465 & 0.23 & 0.941 & 0.033 & 0.479 & 0.223 & 1.028 & 0.059 \\
\hline MELD & 1.074 & 0.988 & 1.167 & 0.092 & 0.962 & 0.818 & 1.130 & 0.634 \\
\hline
\end{tabular}

OR, odds ratio; CI, confidence interval; ALT, alanine aminotransferase; AST, aspartate aminotransferase; Na+, sodium; K+, potassium; INR, international normalized ratio; BR, Biejia-Ruangan; MELD, model for end-stage liver disease.

readmission events in the no-BR group included 3 cases of encephalopathy, 4 cases of HCC, 14 cases of ascites and related spontaneous peritonitis, 11 cases of gastrointestinal bleeding, 3 cases of jaundice, and 1 case of hepatorenal syndrome. When all first hospital readmission events during follow-up were considered, there were significantly more events in the no-BR group, with 36 events ( 0.32 events per patient) vs. 9 events in the BR group ( 0.14 events per patient) $(P=0.01)$. The cumulative incidence of first hospital readmission was significantly higher in the no-BR group than in the BR group $(P=0.004)$ (Figure 3).

Of the 63 patients who were administered with BR, the duration was six months in 26 patients and twelve months in 37 patients. The first hospital readmission events in the BR-6 therapy group included 2 cases of encephalopathy, 1 case of HCC, 2 cases of ascites and related spontaneous peritonitis, and 1 case of gastrointestinal bleeding. The first hospital readmission events in the BR-12 therapy group included 1 
TABLe 4: Univariate and multivariate analyses to predict first hospital readmission in nonalcoholic cirrhosis patients $(N=176)$.

\begin{tabular}{|c|c|c|c|c|c|c|c|c|}
\hline \multirow[t]{2}{*}{ Variable } & \multirow[t]{2}{*}{$\mathrm{HR}$} & \multicolumn{2}{|c|}{$\begin{array}{l}\text { 95\% variable HR CI } \\
\text { for EXP (B) }\end{array}$} & \multirow[t]{2}{*}{$P$ value } & \multirow[t]{2}{*}{ HR } & \multicolumn{2}{|c|}{$\begin{array}{l}\text { 95\% variable HR CI } \\
\text { for EXP (B) }\end{array}$} & \multirow[t]{2}{*}{$P$ value } \\
\hline & & Lower & Upper & & & Lower & Upper & \\
\hline Age (year) & 1.022 & 0.994 & 1.051 & 0.132 & 1.031 & 0.996 & 1.067 & 0.088 \\
\hline ALT & 0.998 & 0.991 & 1.006 & 0.689 & 0.989 & 0.970 & 1.009 & 0.287 \\
\hline AST & 1.001 & 0.998 & 1.003 & 0.649 & 1.008 & 0.994 & 1.022 & 0.276 \\
\hline $\mathrm{Na}$ & 0.911 & 0.842 & 0.986 & 0.021 & 0.963 & 0.882 & 1.051 & 0.395 \\
\hline $\mathrm{K}$ & 2.006 & 0.907 & 4.436 & 0.085 & 1.603 & 0.603 & 4.528 & 0.344 \\
\hline MHE & 6.515 & 3.431 & 12.371 & 0 & 5.805 & 3.007 & 11.206 & 0 \\
\hline Serum albumin $(\mathrm{g} / \mathrm{L})$ & 0.905 & 0.856 & 0.957 & 0 & 0.948 & 0.885 & 1.014 & 0.122 \\
\hline Total bilirubin $(\mu \mathrm{mol} / \mathrm{L})$ & 1.009 & 1.005 & 1.013 & 0 & 1.001 & 0.994 & 1.008 & 0.735 \\
\hline INR & 0.815 & 0.263 & 2.523 & 0.723 & 1.251 & 0.349 & 4.490 & 0.731 \\
\hline Creatinine $(\mu \mathrm{mol} / \mathrm{L})$ & 1.004 & 0.994 & 1.015 & 0.406 & 0.996 & 0.981 & 1.010 & 0.547 \\
\hline White blood cells $\left(10^{9} / \mathrm{L}\right)$ & 0.937 & 0.780 & 1.126 & 0.487 & 0.997 & 0.803 & 1.239 & 0.982 \\
\hline $\mathrm{BR}$ & 0.361 & 0.174 & 0.750 & 0.006 & 0.318 & 0.151 & 0.670 & 0.003 \\
\hline MELD & 1.154 & 1.079 & 1.235 & 0 & 1.145 & 1.068 & 1.227 & 0 \\
\hline
\end{tabular}

HR, hazard ratio; CI, confidence interval; ALT, alanine aminotransferase; AST, aspartate aminotransferase; Na+, sodium; K+, potassium; MHE, minimal hepatic encephalopathy; INR, international normalized ratio; MELD, model for end-stage liver disease; BR, Biejia-Ruangan.

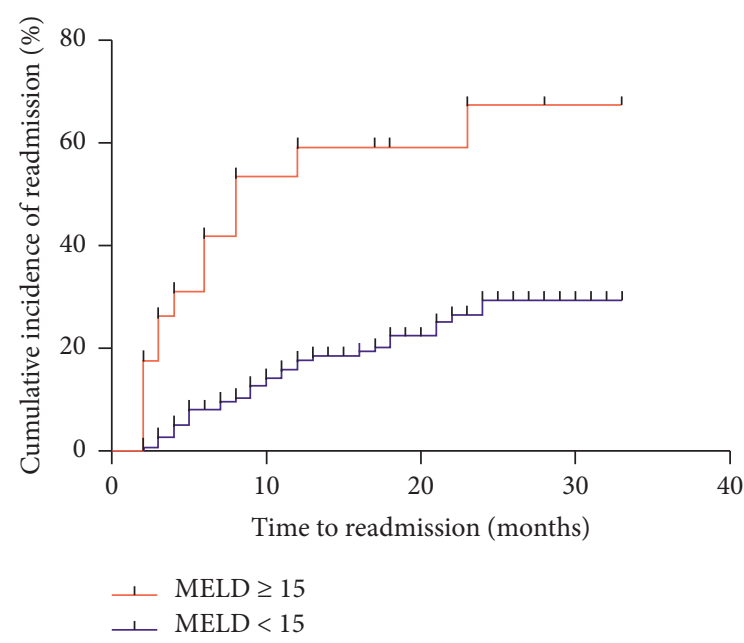

FIGURE 1: There was marked difference in first hospital readmission risk between the MELD $\geq 15$ group and the MELD $<15$ group $(P<0.001)(N=176)$

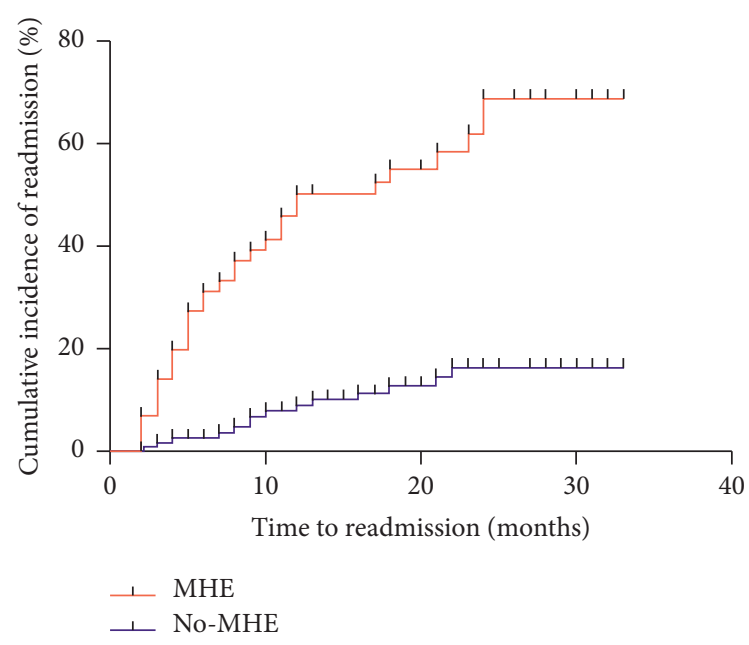

FIgURE 2: The cumulative incidence of first hospital readmission was significantly higher in the MHE group than that in the noMHE group $(P<0.001)(N=176)$.

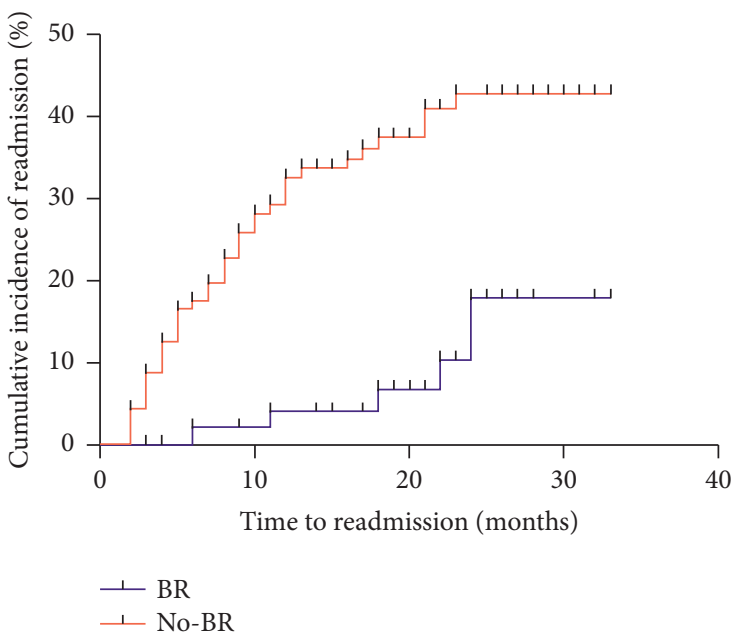

FIgURE 3: The nonalcoholic cirrhotic patients treated with BR were at a lower risk of first hospital readmission than those without BR treatment $(P=0.004 \mathrm{P}=0.004)(N=176)$.

case of ascites and related spontaneous peritonitis and 2 cases of gastrointestinal bleeding. No significant difference was found in the cumulative incidence of first hospital readmission between the BR-6 and BR-12 groups $(P=0.202)$ (Figure 4).

\section{Discussion}

Our study led to three major conclusions. First, a decreased ALB level was related to a higher risk of the presence of $\mathrm{MHE}$ in nonalcoholic cirrhosis patients without previous $\mathrm{OHE}$ or HCC. Second, MHE and the MELD score were risk factors associated with first hospital readmission in nonalcoholic cirrhosis patients. Third, BR helped to decrease the risk of hospital readmission in nonalcoholic cirrhosis patients. No significant difference was observed in the cumulative incidence of first hospital readmission between the groups using BR for 6 months and 12 months. 


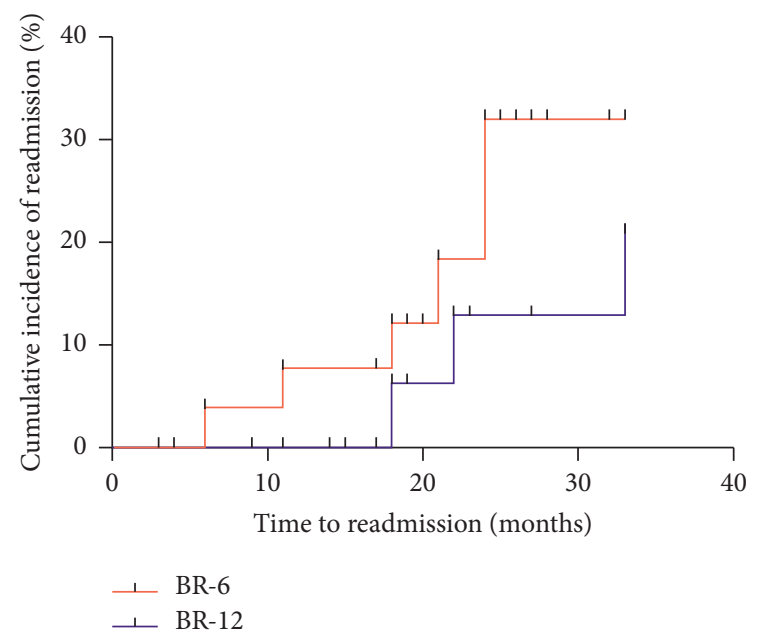

FIGURE 4: No significant difference was found in the cumulative incidence of first hospital readmission between the BR-6 and BR-12 groups $(P=0.202)(N=63)$.

In our study, the prevalence of MHE in nonalcoholic cirrhosis patients was $32.4 \%$, which is lower than that in the general population $[8,28]$. The reason lies in that patients with alcohol-related liver disease were excluded from our study, and alcohol abuse may be a risk factor that affects the results of neuropsychological tests. The effect of alcohol on cognitive function is clear; however, the psychometric alterations in liver cirrhosis patients with MHE due to alcohol abuse are still controversial [7,33-38]. The development of HCC may accelerate the course of the disease at any stage [21], and patients with previous OHE often have worse cognitive function $[7,39,40]$. Therefore, this study excluded patients with alcohol-related liver cirrhosis and previous $\mathrm{OHE}$ and HCC to evaluate the prognosis of patients with liver cirrhosis more precisely.

Among the variables analyzed in this study, the multivariate analysis demonstrated that a decreased ALB level was independently associated with the presence of MHE in nonalcoholic cirrhosis patients. Some studies have concluded that a decreased ALB level may be associated with an increased risk of OHE during hospitalization in cirrhosis patients $[41,42]$. Other studies have reported that the ALB level is an independent risk factor associated with the development of covert hepatic encephalopathy (CHE) [43-45]. In contrast to these studies, our study excluded patients with alcoholic cirrhosis and previous OHE, which may affect the diagnosis of MHE. Moreover, the Child-Pugh classification, which consists of many clinically significant variables in addition to ALB, was also excluded in our multivariate analysis.

MHE is associated with severe liver-related problems and increased mortality. Ampuero et al. [17] suggested that MHE was associated with a reduced 5-year survival rate in patients with liver cirrhosis. Hanai et al. [11] indicated that MHE was associated with an increased risk of mortality in patients with liver cirrhosis, independent of HCC stage or CTP by a propensity score-matching analysis. Barone et al. [46] revealed that the critical flicker frequency (CFF), which is used to diagnose MHE, can be used to predict the mortality risk. Our research concluded that MHE was the most important factor associated with the first hospital readmission of nonalcoholic cirrhosis patients. In our study, we strictly excluded patients with previous OHE and HCC, which may affect the clinical outcome. We drew a conclusion that MHE was closely associated with first hospital readmission in nonalcoholic cirrhosis patients.

The MELD score has been widely applied to assess the severity and prognosis of liver disease. Fung et al. [47] concluded that the MELD score at any time point can accurately predict short-term mortality in patients with severe flares of CHB. Volk et al. [48] found that the predictors of time to first readmission among patients with decompensated cirrhosis included the MELD score, serum Na level, and number of medications at discharge. D'Amico et al. [21] reported that the MELD score was a predictor of long-term survival in patients with decompensated cirrhosis. In our study, the MELD score was also demonstrated to be risk factor associated with the first hospital readmission in nonalcoholic cirrhosis patients.

In recent decades, the role of TCM in the treatment and prevention of fibrosis has been confirmed by a growing number of experiments and clinical studies $[25,49,50]$. BR, which was the first antifibrotic herb approved by the CFDA to treat fibrotic liver disease in China, has been applied for clinical treatment for many years. BR exerts its antifibrotic effect via several mechanisms [51-53]. Recently, a multicenter, randomized, double-blind, placebo-controlled trial showed that addition of BR to nucleoside analog (NAs) in $\mathrm{CHB}$ patients with advanced fibrosis or cirrhosis can improve liver fibrosis regression [54]. Our study found that BR delayed the occurrence of complications of cirrhosis and improved the QOL of nonalcoholic cirrhosis patients without previous OHE or HCC. However, we did not find a significant difference in the cumulative incidence of first hospital readmission between the groups using BR for 6 months and 12 months. Recently, no research has showed the relationship between $\mathrm{BR}$ and $\mathrm{MHE}$. However, a recent report has reported that TCM (Babao Dan) can improve neurocognitive function by targeting and regulating TLR4 inflammatory pathway in MHE patients [55]; a further study about the effect of BR on MHE may be explored.

This study also has several limitations. First, the study was a retrospective cohort study; patients with serious complications are often too weak to take pills, which may lead to bias in clinical characteristics between BR users and non-BR users. Second, this was a single-center study, and the number of patients involved in this study was limited. We expect that multicenter and large-sample randomized controlled trials (RCTs) will be conducted in the future.

In conclusion, MHE is associated with a poor prognosis in nonalcoholic cirrhosis patients. It is essential for doctors to screen for MHE in liver cirrhosis patients using neuropsychological/neurophysiological tests. MHE patients often have an elevated risk of hospital readmission; therefore, it is very important for clinicians to treat MHE patients in a timely manner. BR can decrease the risk of hospital readmission in nonalcoholic cirrhosis patients without previous 
OHE or HCC. BR therapy may play an important role in improving the prognosis of cirrhosis patients, but this finding still needs to be verified by prospective RCTs in the future.

\section{Data Availability}

The data used to support the findings of this study are included within the article.

\section{Conflicts of Interest}

The authors declare that there are no conflicts of interest regarding the publication of this paper.

\section{Acknowledgments}

This work was supported by the National Science Foundation of China (no. 81874435), Application of Clinical Features of Capital City of Science and Technology Commission (no. Z171100001017082), and Beijing Municipal Administration of Hospitals Incubating Program (no. PZ2020030). Thanks are due to all the patients who have underwent the neuropsychological test.

\section{References}

[1] American Association for the Study of Liver Diseases, "European association for the study of the liver. hepatic encephalopathy in chronic liver disease: 2014 practice guideline by the European association for the study of the liver and the American association for the study of liver diseases," Journal of Hepatology, vol. 61, pp. 642-659, 2014.

[2] J. S. Bajaj, K. Saeian, C. M. Schubert et al., "Minimal hepatic encephalopathy is associated with motor vehicle crashes: the reality beyond the driving test," Hepatology, vol. 50, no. 4, pp. 1175-1183, 2009.

[3] J. S. Bajaj, K. Saeian, M. Hafeezullah, R. G. Hoffmann, and T. A. Hammeke, "Patients with minimal hepatic encephalopathy have poor insight into their driving skills," Clinical Gastroenterology and Hepatology, vol. 6, no. 10, pp. 11351139, 2008.

[4] E. Román, J. Córdoba, M. Torrens et al., "Minimal hepatic encephalopathy is associated with falls," American Journal of Gastroenterology, vol. 106, no. 3, pp. 476-482, 2011.

[5] L. Ridola, S. Nardelli, S. Gioia, and O. Riggio, "Quality of life in patients with minimal hepatic encephalopathy," World Journal of Gastroenterology, vol. 24, no. 48, pp. 5446-5453, 2018.

[6] O. Riggio, P. Amodio, A. Farcomeni et al., "A model for predicting development of overt hepatic encephalopathy in patients with cirrhosis," Clinical Gastroenterology and Hepatology, vol. 13, no. 7, pp. 1346-1352, 2015.

[7] R. K. Dhiman, R. Kurmi, K. K. Thumburu et al., "Diagnosis and prognostic significance of minimal hepatic encephalopathy in patients with cirrhosis of liver," Digestive Diseases and Sciences, vol. 55, no. 8, pp. 2381-2390, 2010.

[8] J.-Y. Wang, N. P. Zhang, B. R. Chi et al., "Prevalence of minimal hepatic encephalopathy and quality of life evaluations in hospitalized cirrhotic patients in China," World Journal of Gastroenterology, vol. 19, no. 30, pp. 4984-4991, 2013.
[9] P. Sharma and B. Sharma, "A survey of patterns of practice and perception of minimal hepatic encephalopathy: a nationwide survey in India," Saudi Journal of Gastroenterology, vol. 20, no. 5, pp. 304-308, 2014.

[10] C. Labenz, C. C. Adarkwah, M.-A. Wörns et al., "Management of hepatic encephalopathy in Germany: a survey among physicians," Zeitschrift für Gastroenterologie, vol. 58, no. 1, pp. 49-56, 2020.

[11] T. Hanai, M. Shiraki, S. Watanabe et al., "Prognostic significance of minimal hepatic encephalopathy in patients with liver cirrhosis in Japan: a propensity score-matching analysis," Journal of Gastroenterology and Hepatology, vol. 34, no. 10, pp. 1809-1816, 2019.

[12] D. Gupta, M. Ingle, K. Shah, A. Phadke, and P. Sawant, "Prospective comparative study of inhibitory control test and psychometric hepatic encephalopathy score for diagnosis and prognosis of minimal hepatic encephalopathy in cirrhotic patients in the Indian subcontinent," Journal of Digestive Diseases, vol. 16, no. 7, pp. 400-407, 2015.

[13] J. Ampuero, C. Montoliú, M. Simón-Talero et al., "Minimal hepatic encephalopathy identifies patients at risk of faster cirrhosis progression," Journal of Gastroenterology and Hepatology, vol. 33, no. 3, pp. 718-725, 2018.

[14] J. Fichet, E. Mercier, O. Genée et al., "Prognosis and 1-year mortality of intensive care unit patients with severe hepatic encephalopathy," Journal of Critical Care, vol. 24, no. 3, pp. 364-370, 2009.

[15] A. Bale, C. G. Pai, S. Shetty, G. Balaraju, and A. Shetty, "Prevalence of and factors associated with minimal hepatic encephalopathy in patients with cirrhosis of liver," Journal of Clinical and Experimental Hepatology, vol. 8, no. 2, pp. 156-161, 2018.

[16] S. Rathi, M. Chopra, G. Chouduri et al., "Prevalence of minimal hepatic encephalopathy in patients with liver cirrhosis: a cross-sectional, clinicoepidemiological, multicenter, nationwide study in India: the PREDICT study," Journal of Clinical and Experimental Hepatology, vol. 9, no. 4, pp. 476-483, 2019.

[17] J. Ampuero, M. Simón, C. Montoliú et al., "Minimal hepatic encephalopathy and critical flicker frequency are associated with survival of patients with cirrhosis," Gastroenterology, vol. 149, no. 6, pp. 1483-1489, 2015.

[18] M. M. Lauridsen, O. B. Schaffalitzky de Muckadell, and H. Vilstrup, "Minimal hepatic encephalopathy characterized by parallel use of the continuous reaction time and portosystemic encephalopathy tests," Metabolic Brain Disease, vol. 30, no. 5, pp. 1187-1192, 2015.

[19] S. Montagnese, A. Biancardi, S. Schiff et al., "Different biochemical correlates for different neuropsychiatric abnormalities in patients with cirrhosis," Hepatology, vol. 53, no. 2, pp. 558-566, 2011.

[20] K. R. Patidar, L. R. Thacker, J. B. Wade et al., "Covert hepatic encephalopathy is independently associated with poor survival and increased risk of hospitalization," American Journal of Gastroenterology, vol. 109, no. 11, pp. 1757-1763, 2014.

[21] G. D’Amico, G. Garcia-Tsao, and L. Pagliaro, "Natural history and prognostic indicators of survival in cirrhosis: a systematic review of 118 studies," Journal of Hepatology, vol. 44, no. 1, pp. 217-231, 2006.

[22] A.-P. Lu, H. W. Jia, C. Xiao, and Q.-P. Lu, "Theory of traditional Chinese medicine and therapeutic method of diseases," World Journal of Gastroenterology, vol. 10, no. 13, pp. 1854-1856, 2004.

[23] P. Liu, Y. Y. Hu, C. Liu et al., "Multicenter clinical study on Fuzhenghuayu capsule against liver fibrosis due to chronic 
hepatitis B," World Journal of Gastroenterology, vol. 11, no. 19, pp. 2892-2899, 2005.

[24] J. Qu, Z. Yu, Q. Li et al., "Blocking and reversing hepatic fibrosis in patientswith chronic hepatitis B treated by traditional Chinese medicine (tablets of biejia ruangan or RGT): study protocol for a randomized controlled trial," Trials, vol. 15 , p. $438,2014$.

[25] L. Zhang and D. Schuppan, "Traditional Chinese medicine (TCM) for fibrotic liver disease: hope and hype," Journal of Hepatology, vol. 61, no. 1, pp. 166-168, 2014.

[26] Q. Dong, L.-L. Qiu, C.-E. Zhang et al., "Identification of compounds in an anti-fibrosis Chinese medicine (Fufang Biejia Ruangan pill) and its absorbed components in rat biofluids and liver by UPLC-MS," Journal of Chromatography B, vol. 1026, pp. 145-151, 2016.

[27] P. Ferenci, A. Lockwood, K. Mullen, R. Tarter, K. Weissenborn, and A. T. Blei, "Hepatic encephalopathydefinition, nomenclature, diagnosis, and quantification: final report of the working party at the 11th world congresses of gastroenterology, Vienna, 1998," Hepatology, vol. 35, no. 3, pp. 716-721, 2002.

[28] S.-W. Li, K. Wang, Y. Q. Yu, H.-B. Wang, Y.-H. Li, and J.-M. Xu, "Psychometric hepatic encephalopathy score for diagnosis of minimal hepatic encephalopathy in China," World Journal of Gastroenterology, vol. 19, no. 46, pp. 87458751, 2013.

[29] European Association for the Study of the Liver, "EASL 2017 clinical practice guidelines on the management of hepatitis B virus infection," Journal of Hepatology, vol. 67, pp. 370-398, 2017.

[30] European Association for the Study of the Liver, "EASL clinical practice guidelines: management of hepatitis $\mathrm{C}$ virus infection," Journal of Hepatology, vol. 60, pp. 392-420, 2014.

[31] European Association for the Study of the Liver, European Association for the Study of the Diabetes, and European Association for the Study of the Obesity, "EASL-EASD-EASO clinical practice guidelines for the management of non-alcoholic fatty liver disease," Journal of Hepatology, vol. 64, pp. 1388-1402, 2016.

[32] European Association for the Study of the Liver, "EASL clinical practice guidelines for the management of patients with decompensated cirrhosis," Journal of Hepatology, vol. 69, pp. 406-460, 2018.

[33] C. Shan, S.-Y. Lee, Y.-H. Chang et al., "Neuropsychological functions in han Chinese patients in Taiwan with bipolar II disorder comorbid and not comorbid with alcohol abuse/ alcohol dependence disorder," Progress in Neuro-Psychopharmacology and Biological Psychiatry, vol. 35, no. 1, pp. 131-136, 2011.

[34] C. Guerri and M. Pascual, "Mechanisms involved in the neurotoxic, cognitive, and neurobehavioral effects of alcohol consumption during adolescence," Alcohol, vol. 44, no. 1, pp. 15-26, 2010.

[35] L. E. Fitzpatrick and S. F. Crowe, "Cognitive and emotional deficits in chronic alcoholics: a role for the cerebellum?" The Cerebellum, vol. 12, no. 4, pp. 520-533, 2013.

[36] J. A. Campbell, J. R. Samartgis, and S. F. Crowe, "Impaired decision making on the balloon analogue risk task as a result of long-term alcohol use," Journal of Clinical and Experimental Neuropsychology, vol. 35, no. 10, pp. 1071-1081, 2013.

[37] F. Campagna, S. Montagnese, S. Schiff et al., "Confounders in the detection of minimal hepatic encephalopathy: a neuropsychological and quantified EEG study," Liver International, vol. 35, no. 5, pp. 1524-1532, 2015.
[38] A. Goldbecker, K. Weissenborn, G. Hamidi Shahrezaei et al., "Comparison of the most favoured methods for the diagnosis of hepatic encephalopathy in liver transplantation candidates," Gut Microbiota, vol. 62, no. 10, pp. 1497-1504, 2013.

[39] J. S. Bajaj, L. R. Thacker, D. M. Heuman et al., "The stroop smartphone application is a short and valid method to screen for minimal hepatic encephalopathy," Hepatology, vol. 58, no. 3, pp. 1122-1132, 2013.

[40] J. S. Bajaj, D. M. Heuman, R. K. Sterling et al., "Validation of encephalapp, smartphone-based stroop test, for the diagnosis of covert hepatic encephalopathy," Clinical Gastroenterology and Hepatology, vol. 13, no. 10, pp. 1828-1835, 2015.

[41] Z. Bai, X. Guo, F. Tacke, Y. Li, H. Li, and X. Qi, "Association of serum albumin level with incidence and mortality of overt hepatic encephalopathy in cirrhosis during hospitalization," Therapeutic Advances in Gastroenterology, vol. 12, Article ID $1756284819881302,2019$.

[42] E. B. Tapper, N. D. Parikh, N. Sengupta et al., "A risk score to predict the development of hepatic encephalopathy in a population-based cohort of patients with cirrhosis," Hepatology, vol. 68, no. 4, pp. 1498-1507, 2018.

[43] C. Labenz, G. Toenges, Y. Huber et al., "Development and validation of a prognostic score to predict covert hepatic encephalopathy in patients with cirrhosis," American Journal of Gastroenterology, vol. 114, no. 5, pp. 764-770, 2019.

[44] S. Nardelli, S. Gioia, L. Ridola, A. Farcomeni, M. Merli, and O. Riggio, "Proton pump inhibitors are associated with minimal and overt hepatic encephalopathy and increased mortality in patients with cirrhosis," Hepatology, vol. 70, no. 2, pp. 640-649, 2019.

[45] Y. Zhang, Y. Feng, B. Cao, and Q. Tian, "The effect of small intestinal bacterial overgrowth on minimal hepatic encephalopathy in patients with cirrhosis," Archives of Medical Science, vol. 3, pp. 592-596, 2016.

[46] M. Barone, E. Shahini, A. Iannone et al., "Critical flicker frequency test predicts overt hepatic encephalopathy and survival in patients with liver cirrhosis," Digestive and Liver Disease, vol. 50, no. 5, pp. 496-500, 2018.

[47] J. Fung, L. Y. Mak, A. C. Y. Chan et al., "Model for end-stage liver disease with additional criteria to predict short-term mortality in severe flares of chronic hepatitis B," Hepatology, vol. 72, no. 3, pp. 818-828, 2020.

[48] M. L. Volk, R. S. Tocco, J. Bazick, M. O. Rakoski, and A. S. Lok, "Hospital readmissions among patients with decompensated cirrhosis," American Journal of Gastroenterology, vol. 107, no. 2, pp. 247-252, 2012.

[49] H. Xie, W. Hou, Y. Yang, Y. Yu, F. Wang, and J. Mao, "Effects of Shenqi Neijin powder on activation and apoptosis of hepatic stellate cells in rats with hepatic fibrosis," International Journal of Clinical and Experimental Medicine, vol. 8, no. 2, pp. 2226-2232, 2015.

[50] J. M. Luk, X. Wang, P. Liu et al., "Traditional Chinese herbal medicines for treatment of liver fibrosis and cancer: from laboratory discovery to clinical evaluation," Liver International, vol. 27, no. 7, pp. 879-890, 2007.

[51] J. Zhou, X.-M. Chen, S.-W. Liu, B. Fu, Q. Hong, and S.-J. Wang, "Effects of Biejia Ruangan tablet-containing serum on matrix metalloproteinase- 9 and tissue inhibitor of metalloproteinase-1 expression in cultured renal interstitial fibroblasts," Chinese Journal of Integrative Medicine, vol. 21, no. 2, pp. 152-156, 2015.

[52] F.-R. Yang, B. W. Fang, and J. S. Lou, "Effects of fufang Biejia Ruangan pills on hepatic fibrosisin vivoandin vitro," World Journal of Gastroenterology, vol. 19, no. 32, pp. 5326-5333, 2013. 
[53] S.-G. Guo, W. Zhang, T. Jiang et al., "Influence of serum collected from rat perfused with compound Biejia Ruangan drug on hepatic stellate cells," World Journal of Gastroenterology, vol. 10, no. 10, pp. 1487-1494, 2004.

[54] G. Rong, Y. Chen, Z. Yu et al., "Synergistic effect of BiejiaRuangan on fibrosis regression in patients with chronic hepatitis B treated with entecavir: a multicenter, randomized, double-blinded, placebo-controlled trial," The Journal of Infectious Diseases, 2020.

[55] B. Lu, C. Wu, N. L. B. Azami et al., "Babao Dan improves neurocognitive function by inhibiting inflammation in clinical minimal hepatic encephalopathy," Biomedicine \& Pharmacotherapy, vol. 135, Article ID 111084, 2021. 